\title{
Erratum \\ Erratum to: Stability Properties of the Riemann Ellipsoids
}

\author{
FranCESCO FASSÒ \& DEBRA LEWIS
}

\author{
Communicated by A. MielKe
}

\section{Erratum to: Arch Rational Mech Anal 158 (2001) 259-292 DOI 10.1007/PL00004245}

This paper corrects some numerically based assertions about the regions of Nekhoroshev stability of the Riemann ellipsoids, which appeared in the originally published article.

The conclusions reported in the originally published article regarding the Nekhoroshev stability of the Riemann ellipsoids require correction. In that article, we numerically checked a sufficient condition for Nekhoroshev stability: a property of the fourth-order normal form called "directional quasi-convexity" (DQC). Our conclusion that there is numerical evidence that all spectrally stable Riemann ellipsoids that are nonresonant up to order four are DQC (and hence Nekhoroshev stable) is erroneous.

1. For the ellipsoids of types I, II and III, the conclusion that all nonresonant spectrally stable ellipsoids are DQC was due to a faulty line of code, which controlled the output of the numerical program. New numerical computations, carried out using corrected code, indicate that the 'DQC regions' for the ellipsoids of types I, II and III are significantly smaller than the regions of spectral stability, and have an intricate structure. The newly determined DQC regions are reported in Figs. 1, 2 and 3.

2. Although the coding error discussed in 1. did not affect the computations for the ellipsoids of type $S$ that were reported in the originally published article, in the preparation of this Erratum we applied the DQC test to a much larger number of spectrally stable coparallel $S_{2}$ ellipsoids of unknown Lyapunov stability than was considered in the originally published article. This more detailed numerical investigation indicates a very small set of non-DQC ellipsoids of this type, see

The online version of the original article can be found under doi:10.1007/PL00004245. 


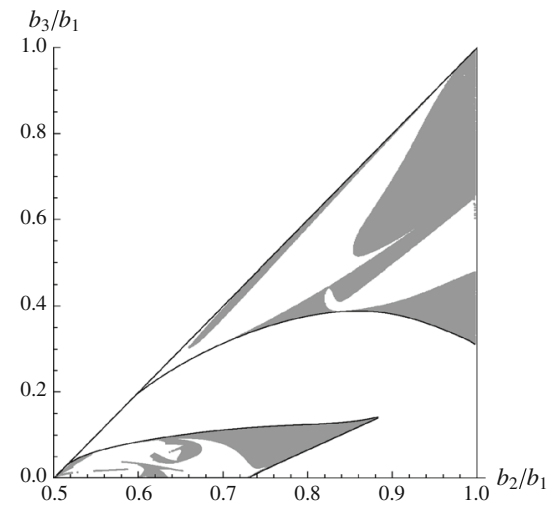

(a)

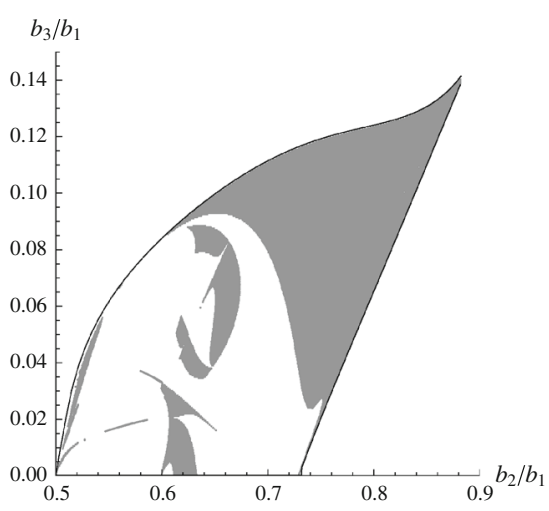

(b)

Fig. 1. a DQC region of the Riemann ellipsoids of type I (shaded). The solid lines denote the boundaries of the existence and spectral stability regions, see Figure 3 of the originally published article. b Enlargement of the lower region

Fig. 4. (The other subfamilies of ellipsoids of type $S$ are either Lyapunov stable or spectrally unstable, and need not be tested.)

In order to obtain a reasonably detailed description of the structure of the DQC regions, the four pictures were constructed by testing for DQC a total of over one million randomly chosen ellipsoids in the regions of spectral stability. Nevertheless, the very small scale structure of these regions, particularly very near the boundaries of the spectral stability regions and near the resonance curves, might merit further investigation. The numerical construction of the normal forms was done using the same algorithms as in the originally published article. For the DQC test we used a new algorithm that was developed in [1]; this algorithm is faster and more reliable than that used in the originally published article in that, avoiding the search of zeros of a function on a circle, reduces the possibility of false positives. All the computations were done using the software package Mathematica.

It should be stressed that a limitation of the figures is that they do not show the curves corresponding to the Riemann ellipsoids whose frequencies satisfy a resonance condition of order up to four (and for which there is a nonzero harmonic in the spectrum of the normal form, see section 5 of the originally published article); for these ellipsoids, the fourth order normal form does not exist and DQC is undefined. There are finitely many such curves, see section 6.1 of the originally published article. Since the normal forms diverge on resonances, we had to exclude from the numerical computations a finite-width band around each of these resonance curves. As in the originally published article we took the size of these bands to be of the order of $10^{-8}$. Establishing how close to resonances points are actually DQC is numerically delicate, and this investigation was not undertaken here. Note however that, even if all nonresonant ellipsoids were DQC, Nekhoroshev stability would in practice be guaranteed only in the complement of 


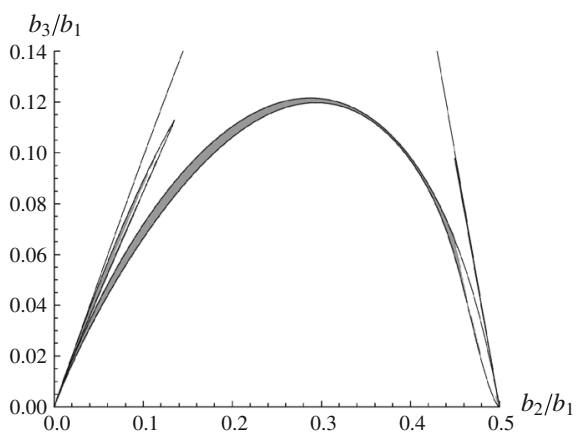

(a)

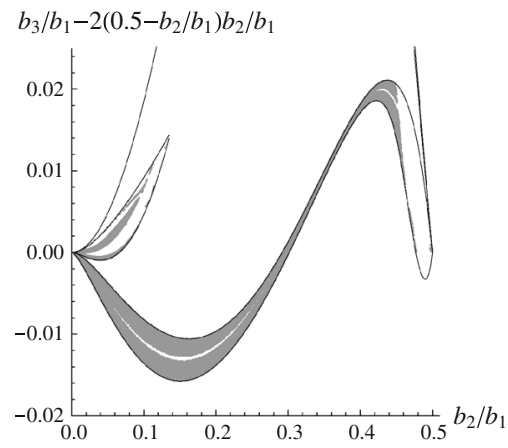

(b)

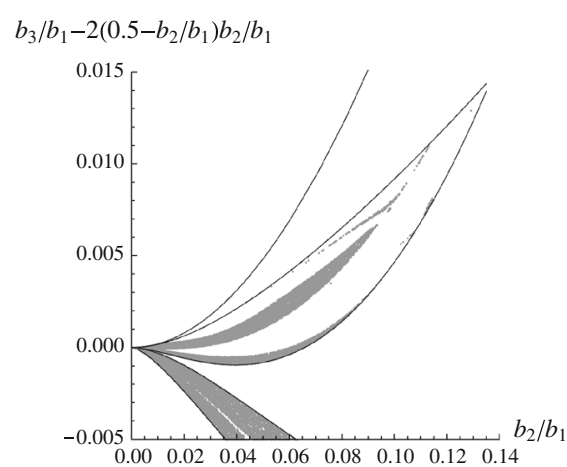

(c)

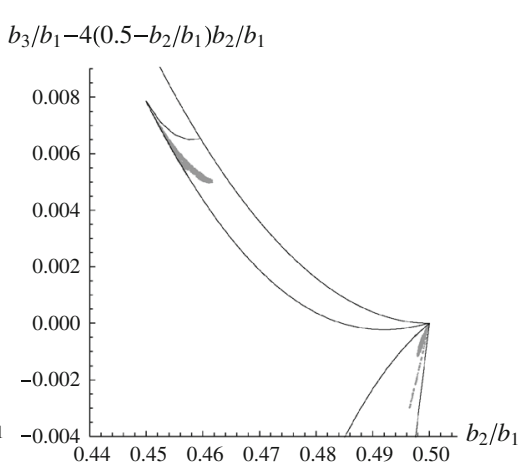

(d)

Fig. 2. DQC region of the Riemann ellipsoids of type II (shaded). The region of spectral stability of these ellipsoids is formed by three narrow fringes, see Figures 3 and 4 of the originally published article, and, in order to show more details of the structure, the vertical component is here plotted relative to the graph of the logistic map $x \mapsto\left(\frac{1}{2}-x\right) s x$, with suitable values of the parameter $s$. The solid lines denote the boundaries of the existence and spectral stability regions. a DQC region, with $s=0$. b DQC region, with $s=2$. c Enlargement of the left fringe, with $s=2$. d Enlargement of the right fringe, with $s=4$

some (small) neighbourhood of the resonance curves. ${ }^{1}$ The relationship between the structure of the DQC region and the resonance curves, if any, would also be of interest.

The conclusion of the investigation initiated in the originally published article is thus numerical evidence for the Nekhoroshev stability of the Riemann ellipsoids only in certain DQC regions.

1 This point was possibly underemphasized in the originally published article. For every fixed distance from a resonance curve, there is a positive threshold $\varepsilon^{*}$ such that Nekhoroshev estimate (21) of the originally published article holds true for $|\varepsilon|<\varepsilon_{*}$, but $\varepsilon^{*}$ goes to zero with the distance from the resonant curve. In practice, one should exclude some band of small but finite width around the resonance curves. 


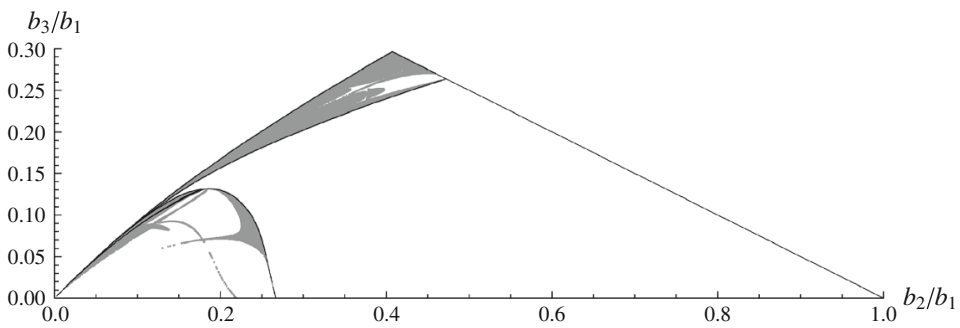

(a)

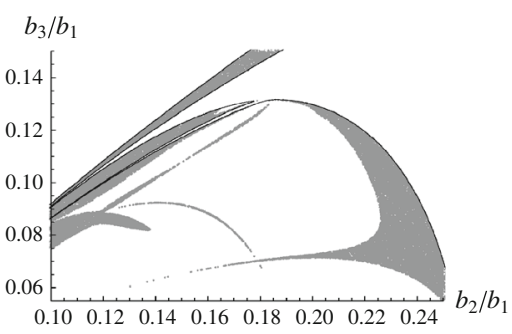

(b)

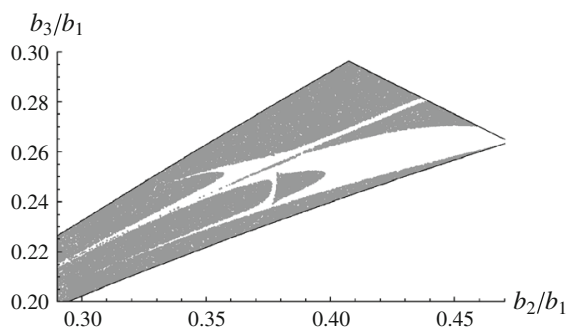

(c)

Fig. 3. a DQC region of the Riemann ellipsoids of type III (shaded). The solid lines denote the boundaries of the existence and spectral stability regions, see Figures 3 and 4 of the originally published article. b, c Enlargements

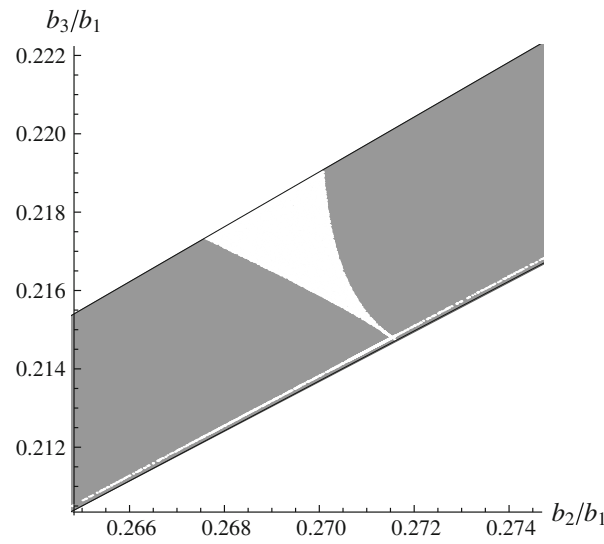

Fig. 4. Detail of the DQC region of the coparallel $S_{2}$ ellipsoids (shaded) in the region $\Sigma_{2}$ consisting of spectrally stable ellipsoids whose Lyapunov stability is unknown. The lower solid line is the boundary of the region of spectral stability and the upper solid line is the boundary of the region of Lyapunov stability (refer to Figure $3 \mathrm{~b}$ of the originally published article). Our current computations indicate that the subsets of $\Sigma_{2}$ not shown in figure consist entirely of DQC ellipsoids, except for a very thin layer near parts of the lower boundary

Since DQC is a sufficient, but not necessary condition for Nekhoroshev stability, the Nekhoroshev stability of the spectrally stable non-DQC ellipsoids is currently unknown. Its determination is of undoubted interest, particularly for the ellipsoids of types I, II and III for which the non-DQC regions are quite extensive. We did 
not undertake its study while preparing this Erratum because it will require new developments in Nekhoroshev theory (see also the Remark on page 283 of the originally published article): a key hypothesis of the Nekhoroshev theorem for nearly integrable systems is a so-called steepness property of a normal form of a sufficiently high order, but a version of the Nekhoroshev theorem for elliptic equilibria satisfying a steepness condition has been proved so far only in a special case with three degrees of freedom; see [2].

\section{References}

1. Dullin, H., Fassò, F.: An algorithm for detecting directional quasi-convexity. BIT Numer. Math. 44, 571-584 (2004)

2. Benettin, G., Fassò, F., Guzzo, M.: Nekhoroshev-stability of L4 and L5 in the spatial restricted three-body problem. Regul. Chaotic Dyn. 3, 56-72 (1998)

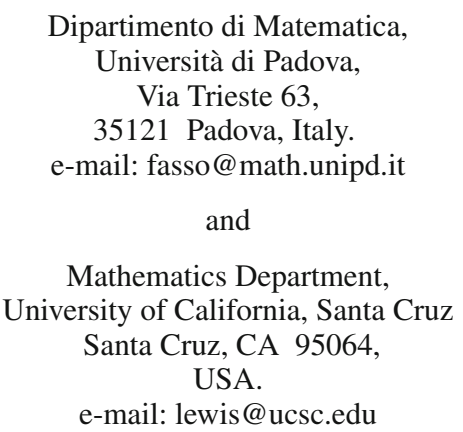

(Received July 11, 2013 / Accepted December 2, 2013)

Published online March 27, 2014 - (C) Springer-Verlag Berlin Heidelberg (2014) 